\title{
Equation invention model and a new built function with numerous interesting properties
}

\author{
Balram Amir Shah : shahba@ced.alliance.edu.in \\ Alliance University: Aerospace Engineering Department
}

\begin{abstract}
This research articulates an approach to invent a substantial equation even without having precise knowledge and skills in mathematics. A model is built such that software will take basic level information and give a complex level equation along with other outputs which make the output equations easier to interpret, visualize and understand its correlations. This expands and amplifies once ability to invent potential equations. Using the model, new eight functions are built and are abbreviated as Couple-Quad Mirror Effect functions (C-Qme function).

It is found that $\left(a+x^{b}\right)^{a-x^{b}}$ is reflex of $\left(a-x^{b}\right)^{a+x^{b}}$ about the $y$-axis line for the odd integer value of $b$. When all eight functions are plotted, any equation always intersects with the other two equation's plot at different points for different values of $a$ and $b$ but the intersection angle always remain at $\pm 45^{\circ}$ from $x$-axis. Integration method refers that, the area under the curve $(a+x)^{a-x}$ and $(a-x)^{a+x}$ are equivalent. The slop of this equation lies one the Euler's number " $e$ " when $x=0$ and on $e^{-1}$ when $a=0$. Paatu function is only one or one of the rarest curves where the same 2-D geometric curve is represented by 8 different functions with only difference in its arithmetic operators of the functions. Also, this is one of the simplest equations to form a beautiful heart geometry.
\end{abstract}

\section{Keywords}

New functions Inventions, mathematical approach, equation computation, multiple equations with equivalent area, Algebra and calculus analysis, different intersection point with same intersection angle, C-Qme Function, Paatu Equation, heart equation.

\section{Introduction}

In the abundance of various kind of equation, there are some equations with astonishing properties which makes it interesting enough to perform a study on such equations and it is a mathematician's 
general interest to be in search of such equations. But there doesn't exist any set of procedures to discover new equations which reduces the difficulties in equation discoveries. In this study, we build a model to invent a random equation from scratch with very basic steps and perform a study on it.

Building a random equation holds the probability that a unique equation may not be unique all the time or in-depth studies are already being performed on that equation. It also holds the probability that many of the built equation will not have an interesting fact. Keeping these facts into consideration, we will adopt a method that will keep the process easy and consume less time to generate multiple in minutes. But in order to build an equation we have to follow many steps including a golden rule of equation development theory which says "without logical reasoning and conditions, an equivalent equation can't be randomly written on every trial but an equivalent function can be written on every trial".

Now we will build a set of procedures and algorithm which will help us to build a new equation. This equation will be analyzed with the help of software and conventional methods to find interesting facts about the equation.

\section{Equation Development Theory}

The objective is to build randomly an equation from scratch. Eg. We can write constants, variable, arithmetic operators, functions, etc in any order and anyhow. But due to the fact that any random equation is not a true mathematical statement every time.

Eg:

$$
\begin{gathered}
5=9 \\
e^{i \pi}=47 \\
\sin (\pi)=\cos (\pi)
\end{gathered}
$$

So, this impedes writing a random equation without logical reasoning but it's not the same for the functions. Any randomly written functions are true mathematical statement and this serves the basic step to build any equations. A function always has variable but not all equations have a variable. But it can be achieved easily as it is easy to convert a variable into a constant.

$\mathrm{Eg}$ : Once the function is built,

$$
f(x)=e^{i y}
$$

With help of conditions and various parameters in the software, it will easily track if $x=-1$ then $y=\pi$. In most of the cases, it may not be straight forward to identify such points but improving and implementing algorithms, it will be easy to find such points in an efficient manner. So, we will get a non-variable equation from a variable equation.

Eg:

$$
e^{i \pi}=-1
$$

Form above argument, we can state that 
Axiom 1: Without logical reasoning and conditions, you can't randomly write an equivalent equation on every trial but you can write an equivalent function on every trial.

Remark 1: It's easy to convert a variable into constant comparatively converting a constant to a variable.

\section{Built Function}

Any function which is used to produce a new function using the concept of mathematics is called Built function. Mathematical concepts may be anything from mathematics (Permutation \& combination, Differentiation, Integration, Functions, methods, etc.). Example: If an existing function is differentiated, it will obtain another unique function in many cases. So, there are many existing functions and there are still more functions yet to be built or discovered which may have an interesting attribute.

These functions can be categories into specific stages for better understanding if it is required. The basic three stages are Built (Parent) function, Ahead function and Branch function.

\section{Definition 1: Mathematical Built Concept}

To build new functions, we will use some random existing mathematics concepts in specific steps. These concepts include Permutation \& combination, Differentiation, Integration, Functions, arithmetic operators, etc.

\section{Definition 2: Built function}

These functions are very basic and smaller compared to its Ahead function. Using various methods of building a new function, Ahead functions are obtained. In various literature, built functions are also referred to as a Parent Function if the function is at its basic level.

\section{Definition 3: Ahead function}

These functions are complex and larger compared to its Built function. It is derived from the Built function. These functions may share similar properties to its Built functions.

\section{Definition 4: Branch function}

One Ahead function is Branch function to another Ahead function if they share a common built function. Any two or more Ahead functions may share similar properties to each other.

\section{First Built function}

In this Research, we will use a function in the form of \pm (constant \pm variable constant $^{\text {co }}$ to build another mathematical function (child function) and perform our further studies on it. This function is termed as First Built function (FB function). 
Eg.

$$
\pm\left(a \pm x^{b}\right)
$$

At certain circumstances, constant $a$ is treated as variable.

Eg.

$$
\pm\left(y \pm x^{b}\right)
$$

\section{Mathematical built function - Hybrid Algorithm}

Terminology used in the algorithm

A constant which acts as a variable and vice versa are termed as convab.

Constants, convab and variables are termed as elements. Brackets and functions are also type of an element.

Positive, negative, multiplication, division, power and root are termed to be Binding operators. These operators bind two or more elements together.

\section{Declaration (Step 1)}

Specify the number of elements with their types.

Eg. (constants : 0 ; convab : 1 ; variables $: 1)$

Note: This algorithm is based on graph analysis and interpretation by humans so maximum 3 variables are optimal to use.

Specify the types of Binding operators to be used. Also, specify their individual numbers.

Eg. (Positive: 2 )

Note: The total number of binding operators is less by one, compared to the total number of elements.

$$
\text { Eg. } 0+a+x^{b}
$$

Coefficient exists due to the multiplication of constants with variable.

The first element is always zero

\section{Formation (Step 2)}

Take the data from the first step and arrange elements and Binding operators in various orders with the help of Permutation. And select an interesting or desired format of a function. 


$$
\operatorname{Eg} \cdot 0+(0+a+x)
$$

Note: In permutation order, the odd position consists of only elements and even positions consist of Binding operators only.

The obtained function in this step is a primary element for the next step. So, this obtained function is termed as a primary element.

\section{Reformation (Step 3)}

In this step, all previous steps are repeated and the primary element must be included in this step. Specify the types and number of elements

$$
\text { Eg. (constants : } 0 \text {; primary element : } 2)
$$

Specify the types and number of Binding operators

$$
\text { Eg. (Power: } 1)
$$

Select the Permutation order

$$
E g \cdot(a+x)^{(a+x)}
$$

\section{Analysis formation (step 4)}

In this step, functions are built and finalized by the analysis process. So, the above obtained function is fed in any analysis or visualization software which makes it easy to interpret. These software are generally Graph tools and spreadsheets.

\section{Eg. (Microsoft Mathematics - Graph tool)}

In this step, every term of the function is manipulated and its data or graphs are obtained. These obtained data and graphs are compared with a different format of function, checked at different conditions \& parameters, etc. Then interesting facts and properties are noted and analyzed more in-depth. Based on results obtained from manipulated function, facts are concluded and again and again same function is manipulated and results are obtained.

\section{Manipulation Tips}

To manipulate the function or equation, below point are considered to make the process easy. But there is no such constrains to anyone to follow below techniques in exact orders.

- Only one Element or binding arithmetic operators are manipulated at a time and results are noted and concluded. Then two changes and so on with the entire new procedure. 
- Binding arithmetic operator's manipulation is preferred first, then the elements.

- The manipulation order of Binding arithmetic operators is followed by difficulty level of understanding and interpretation: Positive < negative $<$ multiplication $<$ division $<$ power $<$ root. And the order of elements is constant $<$ convab $<$ variable $<$ function.

- Inverse of existing Binding arithmetic operators is tested first on the existing Binding arithmetic operator's place rather than other operators in the same position. These inverses are positive-negative, multiplication-division and power-root.

- First, second and third steps of hybrid algorithm can be repeated in any order but introducing new elements should be chosen according to difficulty given by the following order: constants < varient < variable < functions. While adding any new elements, it shouldn't eliminate the primary elements. And this point should be the last choice.

Existing equation is

$$
(a+x)^{(a+x)}
$$

Eg. First operator of first position's primary equation is changed with its inverse.

Therefore, obtained equation is

$$
(a-x)^{(a+x)}
$$

Eg. First operator of second position's primary equation is changed with its inverse.

Therefore, obtained equation is

$$
(a+x)^{(a-x)}
$$

By fixing a as constant $(E g .-1,0,1,2 \ldots .$.$) in above two functions, it is found to have$ similar graphs, so these two equations are noted as it is an interesting fact and further more implementation are done on these functions.

Eg. Negative operator is introduced in first position of operator so the state of the primary element is changed tonegative.

Therefore, the obtained functions are

$$
\begin{aligned}
& (0)-(a-x)^{(a+x)} \\
& (0)-(a+x)^{(a-x)}
\end{aligned}
$$

These two equation also have direct relations with other and with other two previous equations so this equations is also noted 
Eg. Constant $\underline{b}$ is introduced in primary element and if we raise it to an existing variable, then the obtained functions will be

$$
\begin{gathered}
\left(a-x^{b}\right)^{\left(a+x^{b}\right)} \\
\left(a+x^{b}\right)^{\left(a-x^{b}\right)} \\
-\left(a-x^{b}\right)^{\left(a+x^{b}\right)} \\
-\left(a+x^{b}\right)^{\left(a-x^{b}\right)}
\end{gathered}
$$

These four equation also have interesting fact observed on graphs so this equations is also reserved

Since, we have obtained interesting facts about the function which will be covered in coming sections. So, we stop here and finalize it to be main function.

\section{Advantage of Built function}

This allows the mathematician to discover new useful equations with an easy approach.

In many cases, Built, Ahead and Branch function share certain properties and attributes. So, if we are unable to solve an existing equation, then we may find the possibility to get the desired answer or conclusion by solving or relating to its branch or Built function

The Built, Ahead and Branch function may have some common properties, attributes and relation between each other which may be stated as axioms or proved as theorems and formulas.

Since, this function is built-in a sequential way and grouped into family stages, this method will act as an algorithm that will allow the computers to create (generate) new mathematical ideas, concepts, equations, theorems, etc using Mathematical Built Concept. This implies that we can build many potential equations in a short period.

Since this is a type of building an equation in a very sequential way in terms of its Built, Ahead and Branch function along with its number count of variables, constants, convab and binding operators, this will allow us to build hierarchy and family order of an equation. And having hierarchy and family order of an equation may allow us to build an archive for equation in a certain order.

Any existing mathematical equation can be taken and father analyzed, exploring the deeper scope on existing equations.

Ahead function may have multiple built functions that allow us to have an additional correlation about the same equation and to solve the problem easily. 


\section{Limitations}

The algorithm doesn't define the amount of time that will be consumed to discover a new equation and function.

The current set of procedures are not enough to constrain and pose the boundary condition to change the functions into equations in an efficient way.

There is no precise statement and procedure to convert a function into a mathematical formula.

Multiple individuals may end up working on the same equation from various places without knowing each other's work, until the time of publications.

Three dimensional graphs obtained from three variables produces gets difficult to interpret by human and if the function has more than three variables, the usual graph is out of scope to understand the equation and apply the logical reasoning.

\section{C-Qme function}

FB Function is used exponentially to form the Couple Quad Mirror Effect functions (C-Qme function) with variation in the signs of the functions in a certain way to establish the new function which has similar properties. Below are the all Ahead function of FB function.

$$
\begin{aligned}
& f(x)=\left(a+x^{b}\right)^{a-x^{b}} \\
& g(x)=\left(a-x^{b}\right)^{a+x^{b}} \\
& g_{o}(x)=-\left(a-x^{b}\right)^{a+x^{b}} \\
& f_{o}(x)=-\left(a+x^{b}\right)^{a-x^{b}} \\
& f(y)=\left(a+y^{b}\right)^{a-y^{b}} \\
& g(y)=\left(a-y^{b}\right)^{a+y^{b}} \\
& g_{o}(y)=-\left(a-y^{b}\right)^{a+y^{b}} \\
& f_{o}(y)=-\left(a+y^{b}\right)^{a-y^{b}}
\end{aligned}
$$

Equation (1) to (8) are the Ahead function of FB function and these functions are abbreviated as Couple Quad Mirror Effect functions (C-Qme function). In equation (1) to (4), $x$ is an independent variable and in equation (5) to (8), $y$ is an independent variable. So, equation (1) to (4) is abbreviated as $x$ independent function and equation (5) to (8) is abbreviated as $y$ independent function. 
Figure [1]: Plot of equation (1) at the constant's value of $a=1$ and $b=1$.

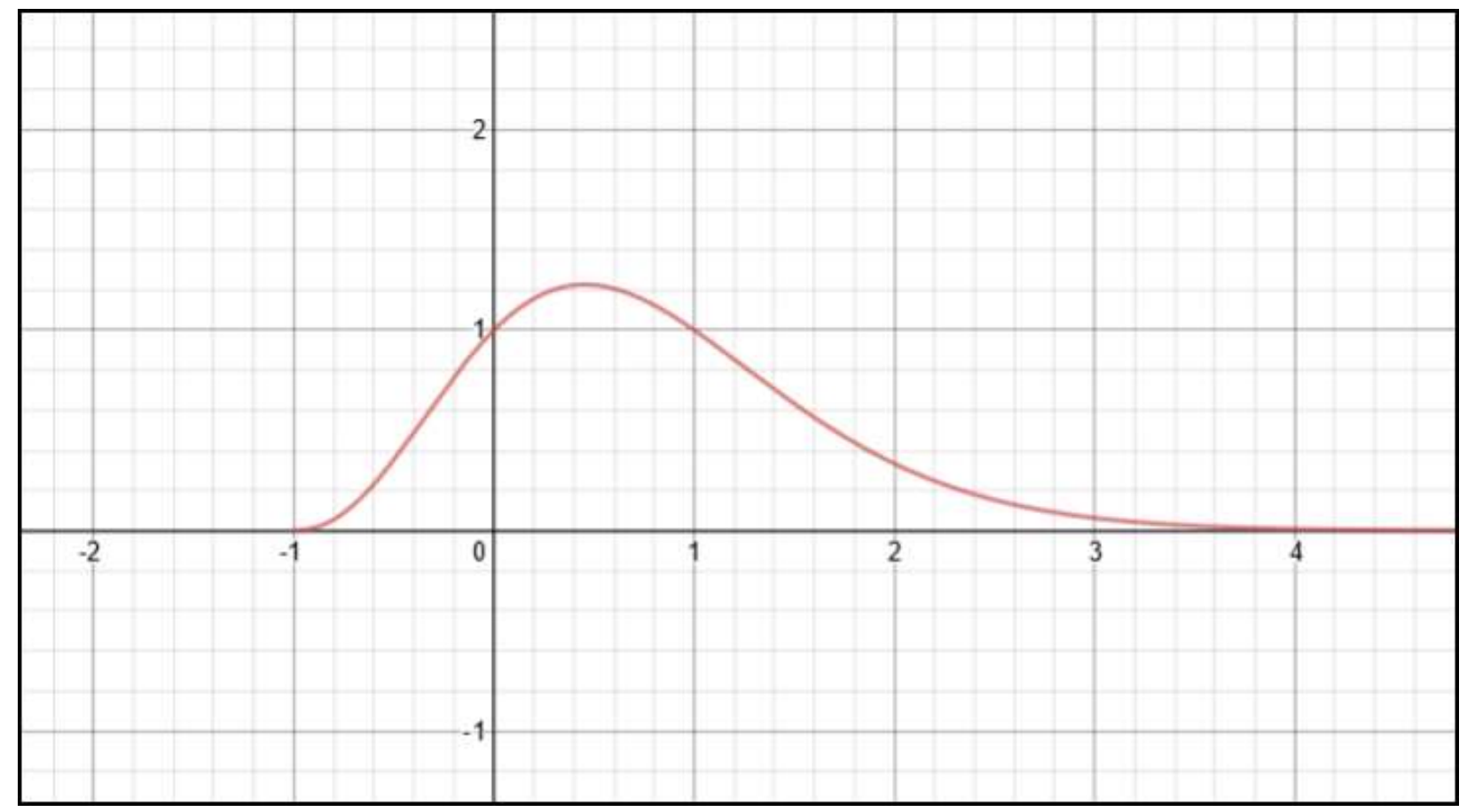

Figure [2]: Plot of (1), (2), (3), \& (4) at the constant's value of $a=1$ and $b=1$.

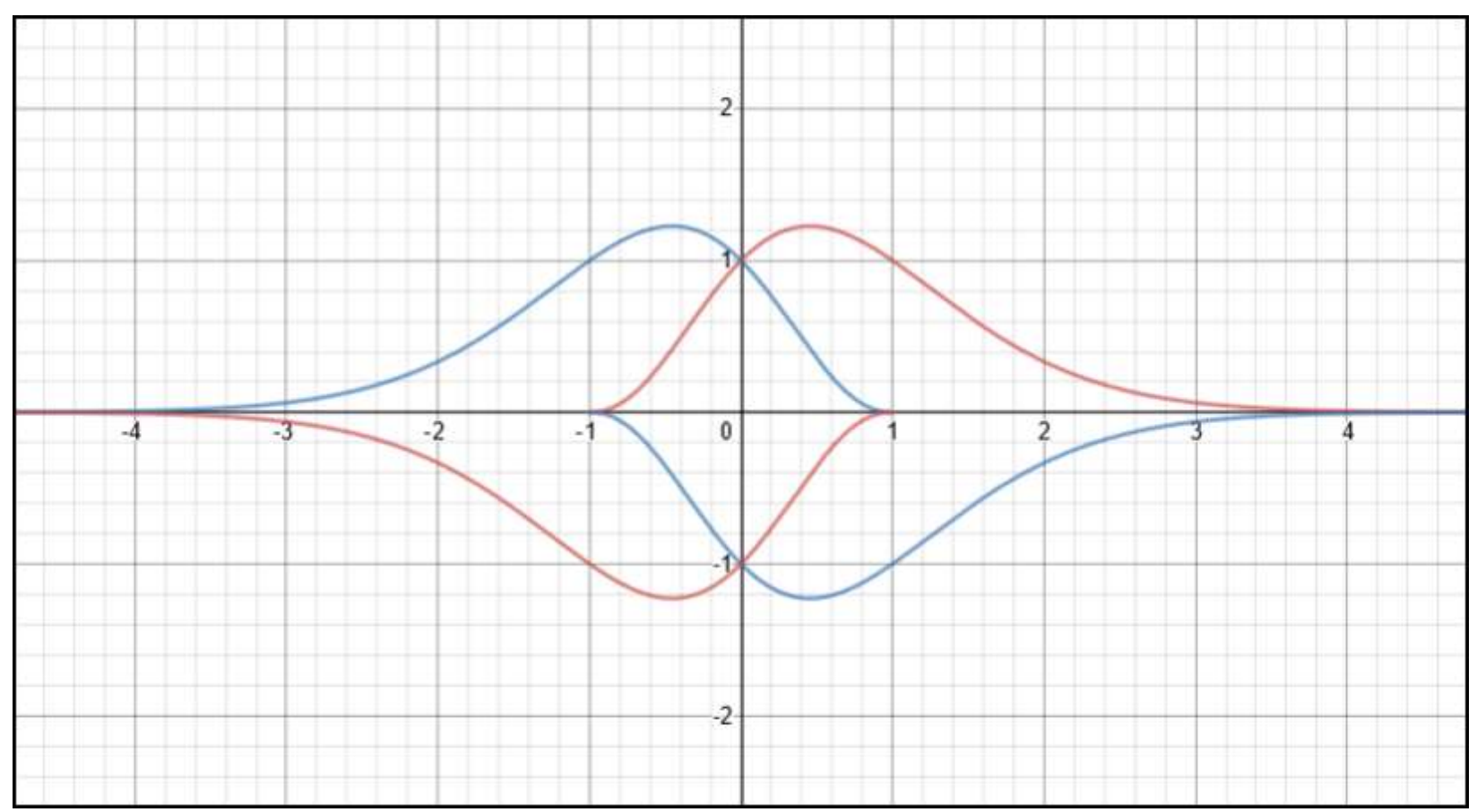

By analyzing the graphs of equation (1) to (4), we observe many similarities in how these functions 
changes as the value of $a$ and $b$ changes. So, based on those observations, the following Postulates are stated.

Postulate 1: If $f(x)=a+x^{b}$ and $g(x)=a-x^{b}$ then $f(x)^{g(x)}$ is reflex of $g(x)^{f(x)}$ on $x$-axis about the $y$-axis line only and for all odd integer value of $b$.

Postulate 2: If $f(x)=a+x^{b}$ then $f(x)^{f(-x)}$ is reflex of $f(-x)^{f(x)}$ on $x-a x i s$ about the $y-$ axis line for all integer value of $b$ and $f(x)^{f(-x)}=f(-x)^{f(x)}$ for even integer value of $b$.

Similarly, equation (5) to (8) are same as equation (1) to (4) except the axis difference. So, postulates (1) and (2) are also applicable for the equation (5) to (8).

Figure [3]: Plot of equation (1), (2), (5) \& (6) at the constant's value of $a=0.8$ and $b=1$.

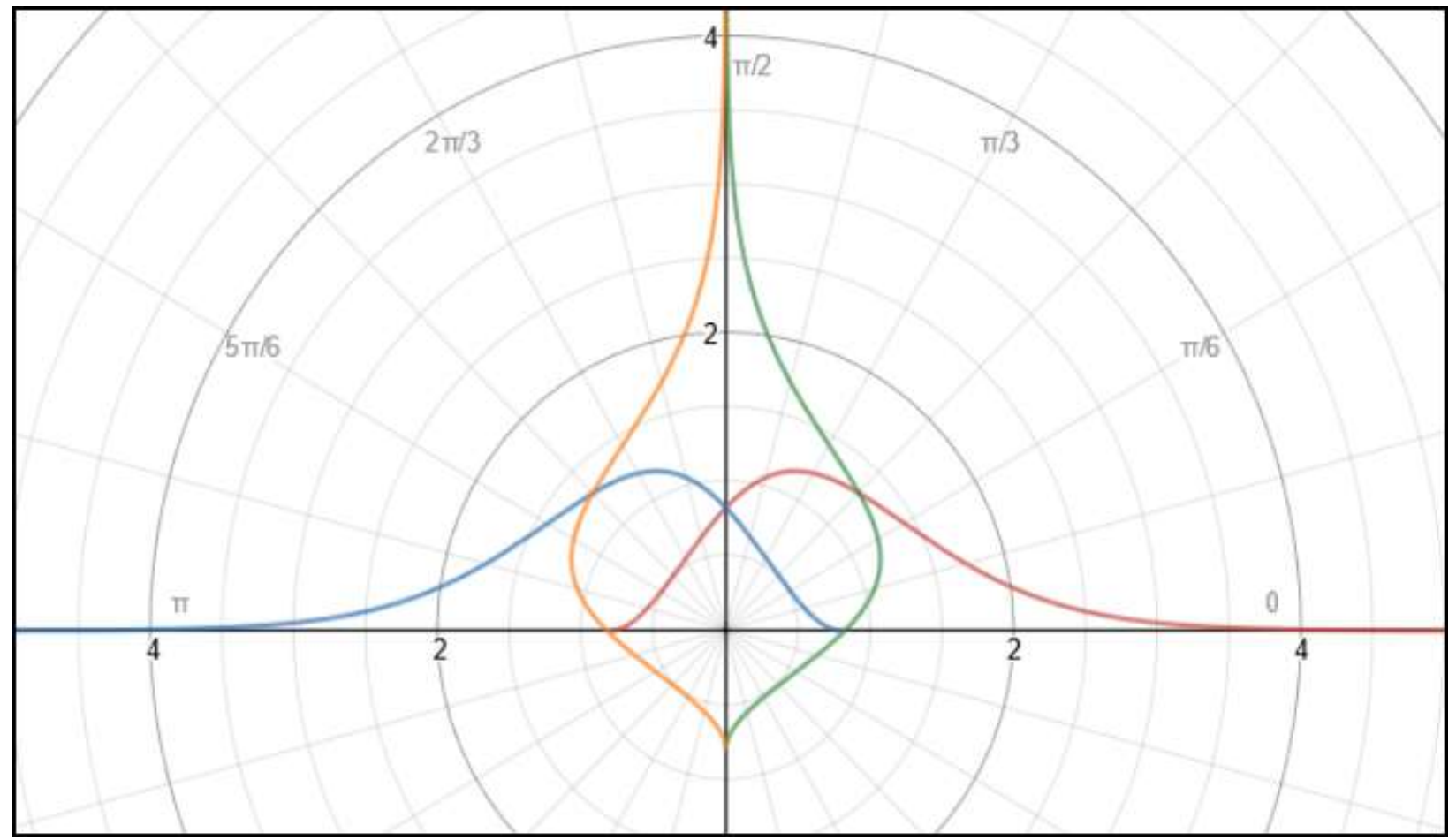

\section{1. $45^{\circ}$ Intersection Point}

When all eight $\mathrm{C}$-Qme functions are plotted, interesting behaviors are observed at the intersection point of $\pm 45^{0}$ from $x$-axis at different conditions which are mentioned below.

Observation 1: At any parameter, $x$ independent function intersects with other $x$ independent function only on $y$-axis line. Similarly, $y$ independent function intersects with other $y$ independent function only on $x$-axis line. 
Observation 2: Every $x$ independent function intersect with the other two unique $y$ independent functions at $\pm 45^{\circ}$ from $x$-axis or $y$-axis line and that intersection point always remains at $\pm 45^{0}$ for any positive integer value $b$ and real number positive value of $a$ while that intersection point varies for different values of $a$ and $b$. Similarly, every $y$ independent function intersects with the other two unique $x$ independent functions at $\pm 45^{0}$ for the same condition as mentioned for $x$ independent function.

Figure [4]: Plot of equation (1), (2), (3), (4), (5), (6), (7), (8) at the constant's value of $a=0.8$ and $b=1$.

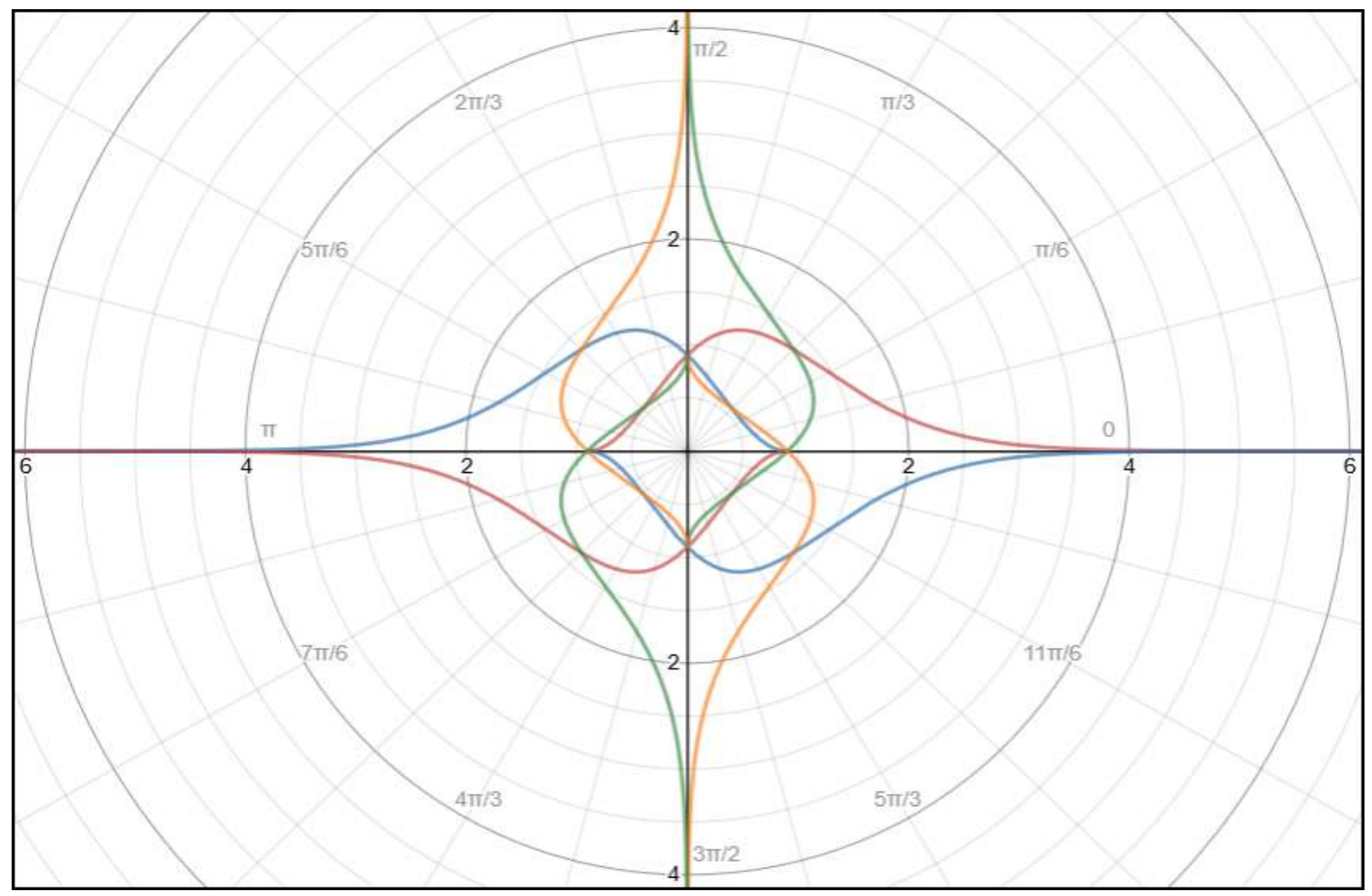

Observation 3: In the second observation, if the value of $a$ is negative real number than the number of intersections occurring at $\pm 45^{0}$ for individual functions varies depending on various conditions as mentioned below.

Observation 3(a): If a function is in the form of $\left(a+x^{b}\right)$ as a base equation while $\left(a-x^{b}\right)$ as its exponent, then for the odd positive integer value of $b$, there occurs only one intersection and for even value of $b$, there occurs two intersections.

Observation 3(b): If the function is in the form of $\left(a-x^{b}\right)$ as a base equation while $\left(a+x^{b}\right)$ as its exponent, then for odd value of $b$, there occurs only one intersection and for even number 
of $b$, intersections are unknown.

Observation 4: At the time of observation 2, when the value of $b$ is a positive integer, there also exist other intersection points which occurs with another 2 unique $y$ independent functions which do not intersect at $\pm 45^{\circ}$ but when the value of $a$ approaches to infinity, the intersection approaches to $\pm 45^{\circ}$ from $x$-axis line.

From the above five observations, a general statement can be stated as a postulate.

Postulate 3: Every individual function of C-Qme function intersects with two of its other functions at different coordinate points for the different positive integer value of $a$ and $b$ but their intersection always remains at $\pm 45^{\circ}$ from $x$-axis line.

Postulate 4: Every individual function of C-Qme function intersect with four of its other functions at $\pm 45^{\circ}$ from $x$-axis when $b$ is a positive integer value and $a$ is equal to infinity from which two intersections always divert from $\pm 45^{\circ}$ when the value of $a$ decreases from infinity.

\section{Paatu function}

In C-Qme function, there are multiple constants which increases the complexity of understanding and solving the equation. For this reason, the calculus studies will be done on C-Qme when the constant value $b$ is one.

Abbreviation 1: When the value of $b$ is unity in C-Qme function then C-Qme function will be abbreviated as Paatu function.

\subsection{Domain}

C-Qme function is an exponential function having a variable in the exponent. So, the function is discontinuous when a variable in the base accounts for a negative value. Eg: let use consider equation (1) when $b$ is unity.

$$
f(x)=(a+x)^{a-x}
$$

So, if

$$
(a+x)<0
$$

Then the function will be discontinous as odd power will yield negative value and even power will yield positive value. Therefore, the domain for the continuous function is defined such that the base equation is always a positive number. This implies the function is continuous when

$$
(a+x) \geq 0
$$

or

$$
x \geq-a
$$


So $a$ is the defining factor at one end of the domain. Similarly, if the base equation for a given function is of type $(a-x), \mathrm{Eg}$

$$
f(x)=(a+x)^{a-x}
$$

then the function is continuous when

$$
(a-x)>0
$$

or

$$
x \geq a
$$

Therefore, if the base equation is $(a+x)$, the domain of the function is between $(-a$ to $\infty)$ on real number line and the domain is $(a$ to $-\infty)$ if the base equation is $(a-x)$.

$$
(a+x) \geq 0
$$

This domain length implies to all Paatu function.

Remark 2: Constant value of $a$ is the soul responsible for one end of the domain for the Paatu function.

Remark 3: The domain of all eight Paatu functions are the same for the same values of $a$.

Remark 4: The domain of any Paatu function is between $(-a$ to $+\infty)$ or $(-\infty$ to $+a)$.

\subsection{Range}

The domain is defined when the base function is a positive value, this implies the range of Paatu function is $(0$ to $+\infty)$. Also, there are functions with negative signs outside the coverage of exponents and for such functions, the domain is $(-\infty$ to 0$)$.

$\mathrm{Eg}$, equation (1), (2), (5) and (6) have a positive initial sign so the range for these equations is between $(0$ to $+\infty)$ and the range for (3), (4), (7) \& (8) is between ( $-\infty$ to 0$)$.

Remark 5: The range of Paatu function is in between of ( 0 to $\pm \infty)$.

\subsection{Y intersection point}

Figure 1 represents the Paatu function. In this figure, $y$-axis always intersects the function at various point for various value of $a$ where $a \in \mathbb{R}$. And the range of these intersections can be found when $x$ approaches to zero

$$
\lim _{x \rightarrow 0} f(x)=\lim _{x \rightarrow 0}(a+x)^{a-x}
$$




$$
\begin{aligned}
& =(a+0)^{a-0} \\
& =a^{a}
\end{aligned}
$$

So, $y$-axis intersects the equation at $a^{a}$

As we know $\infty^{\infty}=\infty$ is undefined so the maximum value of $y$ intersection point is infinity but there exists the lowest value which may be defined.

Let us assume $a$ to be $x$ as a variable. So, the function will be

$$
f(x)=x^{x}
$$

Equation (11) is the $2^{\text {nd }}$ titration and it is defined by other names by different authors, for example: Self-Exponential or couple equation.

$$
f(x)= \begin{cases}\text { continues, } & x \in+\mathbb{R} \\ \text { discontinues, } & x \in-\mathbb{R}\end{cases}
$$

As the function is discontinues for negative numbers, the outcome from the derivative method will fail to account for any properties in that region. Therefore, the outcome of this method is valid only for $x \in+\mathbb{R}$

$$
\frac{d}{d x} f(x)=\frac{d}{d x}\left(x^{x}\right)
$$

Using the Power Rule

$$
\begin{gathered}
=x^{x} \frac{d}{d x}(x \ln (x)) \\
=x^{x}\left(x \frac{d}{d x}(\ln (x))+\ln (x) \frac{d x}{d x}\right) \\
\frac{d}{d x} f(x)=x^{x}(1+\ln (x)) \\
a^{a}(1+\ln (a))=0
\end{gathered}
$$

Therefore, either

$$
a^{a}=0
$$

or

$$
(1+\ln (x))=0
$$

But equation (13) is undefined, so we will use equation (14) to find out the zero of the function

$$
(1+\ln (x))=0
$$




$$
\begin{aligned}
& \ln (x)=-1 \\
& x=e^{-1}
\end{aligned}
$$

Equating (15) into (11) we get

$$
e^{-1\left(e^{-1}\right)}=\frac{1}{e^{1 / e}} \approx 0.6922006
$$

Therefore, any positive number raised to itself will be always greater than $\approx 0.6922006$. This also implies the minimum point of intersection of Paatu function on $y$-axis is $\approx 0.6922 \ldots$.

\subsection{Comparisons of area}

Let us take the functions $f(x)=(a+x)^{a-x}$ and $g(x)=(a-x)^{a+x}$ with its boundary condition. So, the area under this function can be obtained using the close integral within its domain $x \in \mathbb{R}$.

$$
\int_{-a}^{\infty} f(x) d x=\int_{-a}^{\infty}(a+x)^{a-x} d x
$$

Let

Therefore

$$
\begin{gathered}
(a-x)=u \\
x=(a-u) \\
d x=-d u
\end{gathered}
$$

So, when

$$
\begin{array}{ll}
x=-a & ; u=2 a \\
x=\infty \quad & \quad u=-\infty
\end{array}
$$

Therefore

$$
\begin{aligned}
\int_{-a}^{\infty} f(x) d x & =-\int_{2 a}^{-\infty}(a+(a-u))^{u} d u \\
& =-\int_{2 a}^{-\infty}(2 a-u)^{u} d u \\
\int_{-a}^{\infty} f(x) d x & =\int_{-\infty}^{2 a}(2 a-u)^{u} d u
\end{aligned}
$$

Similarly, the area under the function $g(x)$ will be obtained by integrating the function over its domain from $(a$ to $-\infty)$. So, it is expressed as

$$
\int_{-\infty}^{a} g(x) d x=\int_{-\infty}^{a}(a-x)^{a+x} d x
$$


Let

Therefore

So, when

then

Therefore

$$
\begin{aligned}
& (a+x)=v \\
& x=(v-a) \\
& d x=d v
\end{aligned}
$$

$$
\begin{gathered}
x=-\infty \quad ; v=-\infty \\
x=a \quad ; v=2 a
\end{gathered}
$$

Therefore

$$
\begin{aligned}
\int_{-\infty}^{a} g(x) d x & =\int_{-\infty}^{2 a}(a-(v-a))^{v} d v \\
& =\int_{-\infty}^{2 a}(2 a-v)^{v} d v \\
\int_{-\infty}^{a} g(x) d x & =\int_{-\infty}^{2 a}(2 a-v)^{v} d v
\end{aligned}
$$

From equation (16) \&(17) we conclude that

$$
\int_{-a}^{\infty} f(x) d x=\int_{-\infty}^{a} g(x) d x
$$

or

$$
\int_{-a}^{\infty}(a+x)^{a-x} d x=\int_{-\infty}^{a}(a-x)^{a+x} d x
$$

This implies the area under the curve of function (1) and function (2) are equivalent to the unit value of $b$.

As equation (1) is the same as equation (4) with the only difference is in their sign so the magnitude of the area under the curve will same and this implies an area of function (1) is equivalent to the area of function (4). Similarly, the area of function (2) is equivalent to the area of function (3).

As equation (5), (6), (7) and (8) are same as equation (1), (2), (3) and (4) with only difference in variable notation. This implies the area of all the equations are equivalent to each other.

Remark 5 : The area under the Paatu function are equivalent with respect to each other for any given value of $a$.

Postulate 5: The area under $f(x)=(a+x)^{a-x}$ and $g(x)=(a-x)^{a+x}$ are equivalent to any constant value of $a$ within its entire domain of continuous function. 


\section{Elementary Points}

In general, the functions have many interesting points including the zero points but there is no general terminology existing in mathematics literature to convey all these. We will use "elementary points" to convey the interesting point of a function which are basic and general in mathematics.

Definition 5 : Points which are basic points of the function having a general interest to know about these points on any function. These interesting points are as given below:

a) Maxima, minima and saddle points.

b) Inflection points, critical points and zero points at first and second derivative.

c) Points at an integer value of zero, unity and infinity

d) Points associated basics mathematical constants: Euler's number and Archimedes constant.

And these points are called Elementary Points

\subsection{Critical points}

Critical points can be obtained using differential method. So, let $f(x)$ be $y$

Therefore

$$
\begin{gathered}
f(x)=y=(a+x)^{a-x} \\
\ln (y)=\ln (a+x)^{a-x} \\
\frac{d}{d x} \ln (y)=\frac{d}{d x}(a-x) \ln (a+x) \\
\frac{1}{y} \frac{d y}{d x}=\frac{d}{d x}(a-x) \ln (a+x) \\
\frac{d y}{d x}=(a+x)^{a-x}\left(\ln (a+x) \frac{d}{d x}(a-x)+(a-x) \frac{d}{d x}(\ln (a+x))\right) \\
\frac{d y}{d x}=(x+a)^{a-x}\left(\frac{a-x}{a+x}-\ln (a+x)\right)
\end{gathered}
$$

Equation (20) will allow us to state its points at various slops of the function.

$$
\begin{gathered}
\frac{d y}{d x}=(a+x)^{a-x}\left(\frac{a-x}{a+x}-\ln (a+x)\right) \\
=(x+a)^{a-x}\left(\left(\frac{d a}{d x}-\frac{d x}{d x}\right) \ln (a+x)+\frac{a-x}{a+x} \frac{d}{d x}(a+x)\right)
\end{gathered}
$$




$$
\begin{gathered}
=(a+x)^{a-x}\left((0-1) \ln (a+x)+\frac{a-x}{a+x}\left(\frac{d x}{d x}+\frac{d a}{d x}\right)\right) \\
=(x+a)^{a-x}\left(\frac{(1+0)(a-x)}{a+x}-\ln (a+x)\right)
\end{gathered}
$$

when $\frac{d y}{d x}=0 \quad \& \quad a=0$, we get

$$
\begin{gathered}
(0+x)^{0-x}\left(\frac{0-x}{0+x}-\ln (0+x)\right)=0 \\
(x)^{-x}\left(\frac{-x}{x}-\ln (x)\right)=0 \\
\left(\frac{-1-\ln (x)}{(x)^{x}}\right)=0 \\
-1-\ln (x)=0 \\
\ln (x)=-1 \\
x=e^{-1}
\end{gathered}
$$

Therefore, when $x=e^{-1}$

$$
\begin{gathered}
y=(a+x)^{a-x} \\
=\left(0+e^{-1}\right)^{0-e^{-1}} \\
y=e^{e^{-1}}
\end{gathered}
$$

So, when

$$
\frac{d y}{d x}=0 \quad \& \quad x=0
$$

then

$$
\begin{gathered}
(a+x)^{a-x}\left(\frac{a-x}{a+x}-\ln (a+x)\right)=0 \\
(a+0)^{a-0}\left(\frac{a-0}{a+0}-\ln (a+0)\right)=0 \\
(a)^{a}\left(\frac{a}{a}-\ln (a)\right)=0 \\
1-\ln (a)=0 \\
\ln (a)=1 \\
a=e
\end{gathered}
$$

Therefore, at $a=e$

$$
\begin{gathered}
y=(e+0)^{e-0} \\
y=e^{e}
\end{gathered}
$$

So, the critical points for $f(x)$ are 
at

critical points are

and when

the critical points are

$$
\frac{d y}{d x}=0 \quad ; \quad a=0
$$$$
x=e^{-1} \quad \& \quad y=e^{1 / e}
$$$$
\frac{d y}{d x}=0 \quad ; \quad x=0
$$$$
x=e^{-1} \quad \& \quad y=e^{1 / e}
$$

Equation (20) is the first derivative of the function and can be used to find the points at any slop. So, using equation (20), the zero slope of the function can be also expressed as

$$
\frac{a-x}{a+x}=\ln (a+x)
$$

or

$$
e^{a-x}=(a+x)^{a+x}
$$

Equation (25) or (26) has an interesting graph that is related to Euler's number and this equation will be abbreviated as Paatu's slop equation. The right-hand side of the function in equation (26) resembles the self-exponential function which allows us to state

$$
e^{a-x} \geq \frac{1}{e^{1 / e}} \quad \text { if } \quad(a+x) \in+\mathbb{R}
$$

Figure [5]: Graph of Paatu's slope equation. $x$ on the x-axis and on the y-axis.

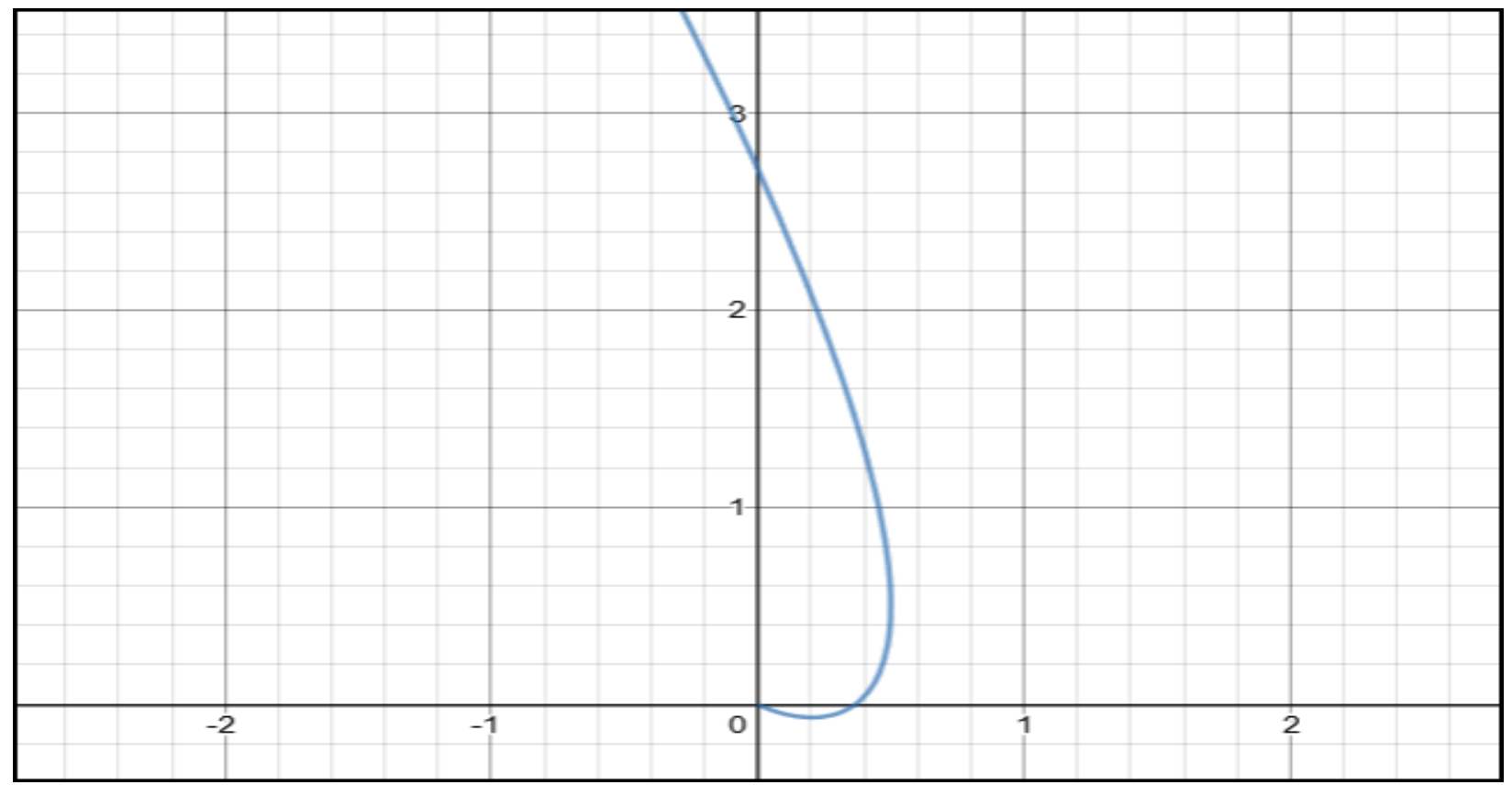

Similarly, the derivative for the function $g(x)$ is 


$$
\frac{d}{d x}(a-x)^{a+x}=(a-x)^{a+x}\left(\ln (a-x)-\frac{a+x}{a-x}\right)
$$

and the critical points for $g(x)$ are

at

$$
\frac{d y}{d x}=0 \quad ; \quad a=0
$$

then critical points are

$$
x=-e^{-1} \quad \& \quad y=e^{1 / e}
$$

and at

$$
\frac{d y}{d x}=0 \quad ; \quad x=0
$$

critical points are at

$$
a=e \quad \& \quad y=e^{e}
$$

The zeros of the function $f(x)$ and $g(x)$ are at $\left(x=e^{-1}, y=e^{1 / e}\right)$ and $\left(x=-e^{-1}, y=e^{1 / e}\right)$ respectively. But considering $a$ as a third variable, both $f(x)$ and $g(x)$ have the same coordinate points for the same condition $\frac{d y}{d x}=0: x=0, y=e^{e}, a=e$ which implies, there is a possibility of identical properties of $f(x)$ and $g(x)$ on the third dimension.

\subsection{Maxima, Minima and Saddle points}

To find the maxima, minima or the saddle point Paatu function, let us consider $a$ as a variable represents of y-axis. This point will allow us to identify the lowest or highest value of $\mathrm{x}$ of the function where slop is zero. So, we will use the partial derivative method to find this point which is given by

$$
D=f_{x x}(x, y) f_{y y}(x, y)+\left(f_{x y}(x, y)\right)^{2}
$$

To proceed with the first and second derivative, let the Qme Function is represented in the equation (30) where variable $x=x$ and variable $a=y$

$$
\begin{gathered}
f=f(x, y)=(y+x)^{(y-x)} \\
\ln (f)=\ln \left((y+x)^{y-x}\right) \\
\frac{\partial}{\partial y}(\ln (f))=\frac{\partial}{\partial y}((y-x) \ln (y+x)) \\
\frac{1}{f} \frac{\partial f}{\partial y}=\frac{\partial}{\partial y}((y-x) \ln (y+x)) \\
\frac{\partial f}{\partial y}=(y+x)^{y-x}\left(\ln (y+x) \frac{\partial}{\partial y}(y-x)+(y-x) \frac{\partial}{\partial y}(\ln (y+x))\right)
\end{gathered}
$$




$$
\begin{gathered}
=(y+x)^{y-x}\left(\ln (y+x)\left(\frac{\partial y}{\partial y}-\frac{\partial x}{\partial y}\right)+\frac{y-x}{y+x} \frac{\partial}{\partial y}(y+x)\right) \\
=(y+x)^{y-x}\left(\ln (y+x)(1-0)+\frac{y-x}{y+x}\left(\frac{\partial y}{\partial y}+\frac{\partial x}{\partial y}\right)\right) \\
=(y+x)^{y-x}\left(\ln (y+x)+\frac{(y-x)(1+0)}{y+x}\right) \\
\frac{\partial f}{\partial y}=(y+x)^{y-x}\left(\ln (y+x)+\frac{y-x}{y+x}\right)
\end{gathered}
$$

Equation (31) is the first derivative of $f(x)$ with respect to $y$.

Second derivative of $f(x)$ with respect $y$ is given by

$$
\begin{aligned}
& \frac{\partial^{2} f}{\partial y^{2}}=\frac{\partial}{\partial y}\left((y+x)^{y-x}\left(\ln (y+x)+\frac{y-x}{y+x}\right)\right) \\
& =\left(\ln (y+x)+\frac{y-x}{y+x}\right) \frac{\partial}{\partial y}\left((y+x)^{y-x}\right)+(y+x)^{y-x} \frac{\partial}{\partial y}\left(\ln (y+x)+\frac{y-x}{y+x}\right) \\
& =\left(\ln (y+x)+\frac{y-x}{y+x}\right)(y+x)^{y-x} \frac{\partial}{\partial y}(\ln (y+x)(y-x))+(y+x)^{y-x}\left(\frac{\partial}{\partial y}(\ln (y+x))+\frac{\partial}{\partial y}\left(\frac{y-x}{y+x}\right)\right) \\
& =(y+x)^{y-x}\left(\ln (y+x)+\frac{y-x}{y+x}\right)\left(\ln (y+x) \frac{\partial}{\partial y}(y-x)+(y-x) \frac{\partial}{\partial y}(\ln (y+x))\right) \\
& +(y+x)^{y-x}\left(\frac{1}{y+x} \frac{\partial}{\partial y}(y+x)+\frac{1}{(y+x)^{2}}\left((y+x) \frac{\partial}{\partial y}(y-x)-(y-x) \frac{\partial}{\partial y}(y+x)\right)\right) \\
& =(y+x)^{y-x}\left(\ln (y+x)+\frac{y-x}{y+x}\right)\left(\ln (y+x)\left(\frac{\partial y}{\partial y}-\frac{\partial x}{\partial y}\right)+\left(\frac{y-x}{y+x}\right) \frac{\partial}{\partial y}(y+x)\right) \\
& +(y+x)^{y-x}\left(\frac{1}{y+x}\left(\frac{\partial y}{\partial y}+\frac{\partial x}{\partial y}\right)+\frac{1}{(y+x)^{2}}\left((y+x)\left(\frac{\partial y}{\partial y}-\frac{\partial x}{\partial y}\right)-(y-x)\left(\frac{\partial y}{\partial y}+\frac{\partial x}{\partial y}\right)\right)\right) \\
& =(y+x)^{y-x}\left(\ln (y+x)+\frac{y-x}{y+x}\right)\left(\ln (y+x)(1-0)+\left(\frac{y-x}{y+x}\right)\left(\frac{\partial y}{\partial y}+\frac{\partial x}{\partial y}\right)\right) \\
& +(y+x)^{y-x}\left(\frac{1+0}{y+x}+\frac{(y+x)(1-0)-(y-x)(1+0)}{(y+x)^{2}}\right) \\
& =(y+x)^{y-x}\left(\ln (y+x)+\frac{y-x}{y+x}\right)\left(\ln (y+x)+\frac{(y-x)(1+0)}{(y+x)}\right)+(y+x)^{y-x}\left(\frac{1}{y+x}+\frac{2 x}{(y+x)^{2}}\right)
\end{aligned}
$$




$$
\begin{aligned}
& =(y+x)^{y-x}\left(\ln (y+x)+\frac{y-x}{y+x}\right)^{2}+(y+x)^{y-x}\left(\frac{1}{y+x}+\frac{2 x}{(y+x)^{2}}\right) \\
& \frac{\partial^{2} f}{\partial y^{2}}=(y+x)^{y-x}\left(\left(\ln (y+x)+\frac{y-x}{y+x}\right)^{2}+\left(\frac{1}{y+x}+\frac{2 x}{(y+x)^{2}}\right)\right)
\end{aligned}
$$

Equation (32) is the second derivative of $f(x)$ with respect to $y$.

First derivative of $f(x)$ the with respect $x$ is given by

$$
\begin{gathered}
f=f(x, y)=(y+x)^{(y-x)} \\
\frac{\partial f}{\partial x}=\frac{\partial}{\partial x}(y+x)^{(y-x)} \\
\ln (f)=\ln \left((y+x)^{y-x}\right) \\
\frac{\partial}{\partial x}(\ln (f))=\frac{\partial}{\partial x}((y-x) \ln (y+x)) \\
\frac{1}{f} \frac{\partial f}{\partial x}=\frac{\partial}{\partial x}((y-x) \ln (y+x)) \\
=(y+x)^{y-x}\left(\ln (y+x) \frac{\partial}{\partial x}(y-x)+(y-x) \frac{\partial}{\partial y}(\ln (y+x))\right) \\
=(y+x)^{y-x}\left(\ln (y+x)\left(\frac{\partial y}{\partial x}-\frac{\partial x}{\partial x}\right)+\frac{y+x}{y+x} \frac{\partial}{\partial x}(y+x)\right) \\
=(y+x)^{y-x}\left(-\ln (y+x)+\frac{y+x}{y+x}(0+1)\right) \\
\frac{\partial f}{\partial x}=(y+x)^{y-x}\left(-\ln (y+x)+\frac{y-x}{y+x}\right)
\end{gathered}
$$

Equation (33) is the first derivative of $f(x)$ with respect to $x$.

Second derivative of $f(x)$ with respect $x$ is given by

$$
\begin{gathered}
\frac{\partial^{2} f}{\partial x^{2}}=\frac{\partial}{\partial x^{2}}\left((y+x)^{y-x}\left(-\ln (y+x)+\frac{y-x}{y+x}\right)\right) \\
=\left(-\ln (y+x)+\frac{y-x}{y+x}\right) \frac{\partial}{\partial x}\left((y+x)^{y-x}\right)+(y+x)^{y-x} \frac{\partial}{\partial x}\left(-\ln (y+x)+\frac{y-x}{y+x}\right) \\
=(y+x)^{y-x}\left(-\ln (y+x)+\frac{y-x}{y+x}\right) \frac{\partial}{\partial x}(\ln (y+x)(y-x))+(y+x)^{y-x}\left(-\frac{\partial}{\partial x}(\ln (y+x))+\frac{\partial}{\partial x}\left(\frac{y-x}{y+x}\right)\right)
\end{gathered}
$$




$$
\begin{aligned}
& =(y+x)^{y-x}\left(-\ln (y+x)+\frac{y-x}{y+x}\right)\left(\ln (y+x) \frac{\partial}{\partial x}(y-x)+(y-x) \frac{\partial}{\partial x}(\ln (y+x))\right) \\
& +(y+x)^{y-x}\left(-\frac{1}{y+x} \frac{\partial}{\partial x}(y+x)+\frac{1}{(y+x)^{2}}\left((y+x) \frac{\partial}{\partial x}(y-x)-(y-x) \frac{\partial}{\partial x}(y+x)\right)\right) \\
& =(y+x)^{y-x}\left(-\ln (y+x)+\frac{y-x}{y+x}\right)\left(\left(\frac{\partial y}{\partial x}-\frac{\partial x}{\partial x}\right) \ln (y+x)+\frac{y-x}{y+x} \frac{\partial}{\partial x}(y+x)\right) \\
& +(y+x)^{y-x}\left(-\frac{1}{y+x}\left(\frac{\partial y}{\partial x}+\frac{\partial x}{\partial x}\right)+\frac{1}{(y+x)^{2}}\left((y+x)\left(\frac{\partial y}{\partial x}-\frac{\partial x}{\partial x}\right)-(y-x)\left(\frac{\partial y}{\partial x}+\frac{\partial x}{\partial x}\right)\right)\right) \\
& =(y+x)^{y-x}\left(-\ln (y+x)+\frac{y-x}{y+x}\right)\left((0-1) \ln (y+x)+\frac{y-x}{y+x}\left(\frac{\partial y}{\partial x}+\frac{\partial x}{\partial x}\right)\right) \\
& +(y+x)^{y-x}\left(-\frac{1+0}{y+x}+\frac{(0-1)(y+x)-(1+0)(y-x)}{(y+x)^{2}}\right) \\
& =(y+x)^{y-x}\left(-\ln (y+x)+\frac{y-x}{y+x}\right)\left(-\ln (y+x)+\frac{(y-x)(1+0)}{(y+x)}\right)+(y+x)^{y-x}\left(-\frac{1}{y+x}-\frac{2 y}{(y+x)^{2}}\right) \\
& =(y+x)^{y-x}\left(\frac{y-x}{y+x}-\ln (y+x)\right)^{2}-(y+x)^{y-x}\left(\frac{1}{y+x}+\frac{2 y}{(y+x)^{2}}\right) \\
& \frac{\partial^{2} f}{\partial x^{2}}=(y+x)^{y-x}\left(\left(\frac{y-x}{y+x}-\ln (y+x)\right)^{2}-\left(\frac{1}{y+x}+\frac{2 y}{(y+x)^{2}}\right)\right)
\end{aligned}
$$

Equation (34) is the second derivative of $f(x)$ with respect to $x$.

Partial double derivative of $f(x)$ the with respect $x$ and $y$ is given by

$$
\begin{gathered}
\frac{\partial^{2} f}{\partial x \partial y}=\frac{\partial}{\partial x}\left(\frac{\partial f}{\partial y}\right) \\
\frac{\partial f}{\partial y}=(y+x)^{y-x}\left(\ln (y+x)+\frac{y-x}{y+x}\right) \\
\frac{\partial^{2} f}{\partial x \partial y}=\frac{\partial}{\partial x}\left((y+x)^{y-x}\left(\ln (y+x)+\frac{y-x}{y+x}\right)\right) \\
=\left(\ln (y+x)+\frac{y-x}{y+x}\right) \frac{\partial}{\partial x}\left((y+x)^{y-x}\right)+(y+x)^{y-x} \frac{\partial}{\partial x}\left(\ln (y+x)+\frac{y-x}{y+x}\right) \\
=(y+x)^{y-x}\left(\ln (y+x)+\frac{y-x}{y+x}\right)\left(\ln (y+x) \frac{\partial}{\partial x}(y-x)+(y-x) \frac{\partial}{\partial x}(\ln (y+x))\right)
\end{gathered}
$$




$$
\begin{aligned}
& +(y+x)^{y-x}\left(\frac{1}{y+x} \frac{\partial}{\partial x}(y+x)+\frac{1}{(y+x)^{2}}\left((y+x) \frac{\partial}{\partial x}(y-x)-(y-x) \frac{\partial}{\partial x}(y+x)\right)\right) \\
= & (y+x)^{y-x}\left(\ln (y+x)+\frac{y-x}{y+x}\right)\left(\ln (y+x)\left(\frac{\partial y}{\partial x}-\frac{\partial x}{\partial x}\right)+\frac{y-x}{y+x} \frac{\partial}{\partial x}(y+x)\right) \\
& +(y+x)^{y-x}\left(\frac{1}{y+x}\left(\frac{\partial y}{\partial x}+\frac{\partial x}{\partial x}\right)+\frac{1}{(y+x)^{2}}\left((y+x)\left(\frac{\partial y}{\partial x}-\frac{\partial x}{\partial x}\right)-(y-x)\left(\frac{\partial y}{\partial x}+\frac{\partial x}{\partial x}\right)\right)\right) \\
= & (y+x)^{y-x}\left(\ln (y+x)+\frac{y-x}{y+x}\right)\left((0-1) \ln (y+x)+\frac{y-x}{y+x}\left(\frac{\partial y}{\partial y}+\frac{\partial y}{\partial y}\right)\right) \\
=(y+x)^{y-x} & \left(\ln (y+x)+\frac{y-x}{y+x}\right)\left(-\ln (y+x)+\frac{(1+0)(y-x)}{(y+x)}\right)+(y+x)^{y-x}\left(\frac{1}{y+x}-\frac{2 y}{(y+x)^{2}}\right) \\
= & (y+x)^{y-x}\left(\ln (y+x)+\frac{y-x}{y+x}\right)\left(-\ln (y+x)+\frac{(0-1)(y+x)-(1+0)(y-x)}{y+x}\right)+(y+x)^{y-x}\left(\frac{1}{y+x}-\frac{2 y}{(y+x)^{2}}\right) \\
& \frac{\partial^{2} f}{\partial x \partial y}=(y+x)^{y-x}\left(\left(\frac{y-x}{y+x}\right)^{2}-(\ln (y+x))^{2}+\frac{1}{y+x}-\frac{2 y}{(y+x)^{2}}\right)
\end{aligned}
$$

Equation (35) is a partial double derivative of the function $f(x)$ with respect $x$ and $y$.

So, to find the maximum or minimum point of $a$ on $y$-axis and $x$, let us find the values of $x$ and $y$

$$
\begin{aligned}
& f_{x}=(y+x)^{y-x}\left(-\ln (y+x)+\frac{y-x}{y+x}\right) \\
& f_{y}=(y+x)^{y-x}\left(\ln (y+x)+\frac{y-x}{y+x}\right)
\end{aligned}
$$

Adding equation (36) and (37) when $f_{x}$ and $f_{y}$ is zero, we get

$$
(y+x)^{y-x} 2\left(\frac{y-x}{y+x}\right)=0
$$

This implies either

$$
(y+x)^{y-x}=0
$$

or

$$
\frac{y-x}{y+x}=0
$$


Since $(y+x)^{y-x}=0$ is undefined

And

$$
\begin{aligned}
& \frac{y-x}{y+x}=0 \\
& y-x=0
\end{aligned}
$$

Therefore

$$
x=y
$$

Substituting $x=y$ in equation (38), we obtain

$$
\begin{gathered}
(x+x)^{x-x}\left(\ln (x+x)+\frac{x-x}{x+x}\right)=0 \\
1^{0}(\ln (2 x)+0)=0 \\
2 x=e^{0} \\
x=\frac{1}{2} \\
y=\frac{1}{2} \\
f(x, y)=(y+x)^{(y-x)} \\
=(0.5+0.5)^{(0.5-0.5)} \\
f(x, y)=1
\end{gathered}
$$

Substituting equation (39) and (40) in equation (32), (34) and (35), we obtain

$$
\begin{aligned}
& f_{y y}=(y+x)^{y-x}\left(\left(\ln (y+x)+\frac{y-x}{y+x}\right)^{2}+\left(\frac{1}{y+x}+\frac{2 x}{(y+x)^{2}}\right)\right) \\
& =(0.5+0.5)^{0.5-0.5}\left(\left(\ln (0.5+0.5)+\frac{0.5-0.5}{0.5+0.5}\right)^{2}+\left(\frac{1}{0.5+0.5}+\frac{2(0.5)}{(0.5+0.5)^{2}}\right)\right) \\
& =1\left((0+0)^{2}+(1+1)\right) \\
& f_{y y}=2
\end{aligned}
$$




$$
\begin{aligned}
& f_{x y}=(y+x)^{y-x}\left(\left(\frac{y-x}{y+x}\right)^{2}-(\ln (y+x))^{2}+\frac{1}{y+x}-\frac{2 y}{(y+x)^{2}}\right) \\
& =(0.5+0.5)^{0.5-0.5}\left(\left(\frac{0.5-0.5}{0.5+0.5}\right)^{2}-(\ln (0.5+0.5))^{2}+\frac{1}{0.5+0.5}-\frac{2(0.5)}{(0.5+0.5)^{2}}\right) \\
& =1(0-0)+1-1) \\
& f_{x y}=0 \\
& f_{x x}=(y+x)^{y-x}\left(\left(\frac{y-x}{y+x}-\ln (y+x)\right)^{2}-\left(\frac{1}{y+x}+\frac{2 y}{(y+x)^{2}}\right)\right) \\
& =(0.5+0.5)^{0.5-0.5}\left(\left(\frac{0.5-0.5}{0.5+0.5}-\ln (0.5+0.5)\right)^{2}-\left(\frac{1}{0.5+0.5}+\frac{2(0.5)}{(0.5+0.5)^{2}}\right)\right) \\
& =1\left((0-0)^{2}-(1+1)\right) \\
& f_{x x}=-2
\end{aligned}
$$

Using equation (42), (43) and (44) in (29), we obtain

$$
\begin{gathered}
D=f_{x x}(x, y) f_{y y}(x, y)+\left(f_{x y}(x, y)\right)^{2} \\
D=2(-2)+(0)^{2} \\
D=-4
\end{gathered}
$$

Similarly, the first, second and partial double derivative for the function $g(x, y)$ are

$$
\begin{gathered}
g_{x}=(y-x)^{y+x}\left(\ln (y-x)-\frac{y+x}{y-x}\right) \\
g_{y}=(y-x)^{y+x}\left(\ln (y-x)+\frac{y+x}{y-x}\right) \\
g_{y y}=(y-x)^{y+x}\left(\left(\ln (y-x)+\frac{y+x}{y-x}\right)^{2}+\left(\frac{2}{y-x}-\frac{y+x}{(y-x)^{2}}\right)\right) \\
g_{x y}=(y-x)^{y+x}\left((\ln (y-x))^{2}-\left(\frac{y+x}{y-x}\right)^{2}+\frac{2 y}{(y-x)^{2}}-\frac{1}{y-x}\right) \\
g_{x x}=(y-x)^{y+x}\left(\left(\ln (y-x)-\frac{y+x}{y-x}\right)^{2}-\left(\frac{2}{y-x}+\frac{y+x}{(y+x)^{2}}\right)\right)
\end{gathered}
$$

The point of interest is obtained by subtracting equation (47) from equation (46). 


$$
\begin{gathered}
0=(y-x)^{y+x}\left(\ln (y-x)-\frac{y+x}{y-x}\right)-(y-x)^{y+x}\left(\ln (y-x)+\frac{y+x}{y-x}\right) \\
0=(y-x)^{y+x}\left(-2 \frac{y+x}{y-x}\right)
\end{gathered}
$$

Therefore

$$
(y-x)^{y+x}=0
$$

or

$$
\begin{gathered}
\frac{y+x}{y-x}=0 \\
y+x=0 \\
x=-y
\end{gathered}
$$

Substituting equation (51) in equation (46), we obtain

$$
\begin{gathered}
(y+y)^{y-y}\left(\ln (y+y)-\frac{y-y}{y+y}\right) \\
\ln (2 y)=0
\end{gathered}
$$

therefore

$$
y=\frac{1}{2}
$$

and

$$
x=-\frac{1}{2}
$$

also

$$
\begin{gathered}
f(x, y)=(y-x)^{(y+x)} \\
=(0.5-(-0.5))^{(0.5+0.5)} \\
f(x, y)=1
\end{gathered}
$$

To identify the points are maxima, minima or saddle point, let us substitute obtained points (52) and (53) in (48), (49) and (50).

$$
\begin{aligned}
& g_{x x}=-2 \\
& g_{y y}=2
\end{aligned}
$$




$$
g_{x y}=0
$$

Substituting (55), (56) and (57) the obtained value in (29) we obtain

$$
\begin{gathered}
D=g_{x x}(x, y) g_{y y}(x, y)+\left(g_{x y}(x, y)\right)^{2} \\
D=2(-2)+(0)^{2} \\
D=-4
\end{gathered}
$$

As $D$ value is negative in equation (45) and (58), this implies the obtained elementary point is a saddle point for both the function.

Assuming the value of $x=x, y=a$ and $f(x, y)=y$ from the assumption taken to derivative the function in standardized notations, function $f(x)$ and $g(x)$ have saddle points of $(x=0.5 ; y=1 ; a=0.5)$ and $(x=-0.5 ; y=1 ; a=0.5)$ respectively. This implies the lowest zero slop for the Paatu functions is at $a=0.5$.

\section{Results}

Taking facts and statements into consideration, a model is developed to build an equation. With this model, C-Qme function is obtained with numerous properties as mentioned below

- When $f(x)=a+x^{b}$ and $g(x)=a-x^{b}$ then $f(x)^{g(x)}$ is reflex of $g(x)^{f(x)}$ on $x$-axis about the $y$-axis line only and for all odd integer value of $b$.

- When $f(x)=a+x^{b}$ then $f(x)^{f(-x)}$ is reflex of $f(-x)^{f(x)}$ on $x$-axis about the $y$-axis line for all integer value of $\mathrm{b}$ and $f(x)^{f(-x)}=f(-x)^{f(x)}$ for even integer value of $b$.

- Every individual function of C-Qme function intersect with four of its other functions at $\pm 45^{0}$ from $x$-axis when $b$ is a positive integer value and $a$ is equal to infinity from

which two intersections always divert from $\pm 45^{\circ}$ when the value of $a$ decreases from infinity.

- Every individual function of C-Qme function intersect with two of its other functions at different coordinate points for the different positive integer value of $a$ and $b$ but their intersection always remains at $\pm 45^{\circ}$ from $x$-axis line. 
- The area under $f(x)=(a+x)^{a-x}$ and $g(x)=(a-x)^{a+x}$ are equivalent to any constant value of $a$ within its entire domain of a continuous function.

The Paatu function $f(x)=(a+x)^{a-x}$ yield zero slop point at $a=e$ when $x=0$ and at $x=e^{-1}$ when $a=0$. In general, the zero of function is given by Paatu's equation.

The lowest zero slop of the Paatu function is at $x= \pm 0.5, y=1 \& a=0.5$.

\section{Discussion}

The build algorithm is just at the initial stage and can be developed with a better algorithm to yield higher and quality outputs. Developing a specific software will allow us to get the output in less time as well as with less mind pressure and intelligence.

The graphs for all Paatu Functions are identical, it allows us to state all related results with just one equation. Eg: critical points, intersection points, the area under the curve, etc.

Paatu functions zero slop is also related to Euler number but the equation which defines the zero slop cannot be simplified further which implies it is at its basic form and something can be derived from that equations.

Since C-Qme functions have intersection angle at $\pm 45^{\circ}$ even though its intersection point always varies, this property is seen for the equation of a line which forms a square. $\mathrm{Eg}$

$$
\begin{aligned}
& f(x)=+a \\
& f(x)=-a \\
& f(y)=+a \\
& f(y)=-a
\end{aligned}
$$

This function will form square and the intersection will at $\pm 45^{\circ}$ but intersection point will be different for different values of $a$.

So, in section $7,8, \& 9$, we have elaborated some of the methods applied to get some interesting facts about the developed equation. These methods can also be build and solved using software which will make the task complete in minutes.

\section{Future Works}

The step of algorithms can be converted to build the software that will improve the equation analysis workload drastically. 
As equation building theory is built from very basic level, we can introduce a separate journal or archive which holds the hierarchy system of all equations and the advantages will be numerous. Hierarchy of the below equation can be introduced by assigning the numbers

$$
\left(+a+x^{+b}\right)^{\left(+a-x^{+b}\right)}
$$

Number of elements (Constants : Variables $)=(2 \times 2: 1 \times 2)$

Number of binding operators (Positive : Negative : Multiplication : Division : Power : Roots)

$$
=(5: 1: 0: 0: 1: 0)
$$

More conditions can be done to increase the accuracy to define the category of the equation: Example: combination order, etc.

\section{Conclusion}

Investigation into the scope to find new ways to invent equation, model and algorithm is developed to build new and potential equation/function. Using this model, an equation is built with numerous attributes, it is C-Qme function. All the various facts were elaborated in the entire section.

Paatu function have eight various functions which represent the same curve on the graph without changing any elements and position of elements of the equation except the operators.

\section{Acknowledgment}

I would like to thanks Ms. Arpita Manju Roddanavar who helped me to be a good writer due to which it was possible for me to write this paper. She brought a situation that made me to develop an algorithm to enhance and accelerate anyone's creativity in equation development in mathematics.

I also like to put forward, the calculations and graph plotting were made easy due to the use of Microsoft calculator 4.0, an online graph by desmos.com and a derivative online calculator by David Scherfgen IT Services. 